\title{
Quantitative Exposure Assessment of Enterotoxin-Producing Staphylococcus aureus in Chicken Briyani
}

\author{
P. Selvan* \\ Department of Food and Industrial and Microbiology, College of Food and Dairy \\ Technology, Koduvalli, Chennai - 600 052, Tamil Nadu, India \\ *Corresponding author
}

\begin{tabular}{l} 
Ke y w o r d s \\
Enterotoxin- \\
Producing $S$. \\
aureus, Quantitative \\
Exposure \\
Assessment, \\
Chicken Briyani \\
\hline Article Info \\
\hline Accepted: \\
17 March 2019 \\
Available Online: \\
10 April 2019
\end{tabular}

Keywords

A Quantitative Risk Assessment Model was constructed to estimate the probability that a serving of Chicken Briyani prepared in the catering units at Tamil nadu, India is contaminated with $S$. aureus. Existing data, predictive models and user input were used to define the input settings. The retail to table pathway was modeled as a series of nodes and associated pathogen events that included initial contamination of raw chicken at retail, growth during caterer transport, storage at catering establishments, thermal inactivation during cooking, post process contamination during serving (on the day consumption) and storage of leftover (consumption after overnight storage). The extent of S. aureus growth in each node (with exceptions) were assessed using Combase predictive models whereas thermal inactivation during cooking was quantified using simple mathematical calculations as there was no suitable inactivation model for $S$. aureus. The data, models and user inputs were entered into an Excel (Microsoft, Redmond, WA) spreadsheet. Modeling @RISK software (Palisade Corporation) was used to perform Monte Carlo simulation of $\sim 10000$ iterations. Results of exposure assessment and probability of illness revealed that $36.67 \%$ of the population consuming chicken briyani is at risk of staphylococcal food intoxication (at both node 5\&6). In $95 \%$ of the cases, the probability of getting illness due to the consumption of $S$. aureus contaminated Chicken Briyani to at least one recipient is equal to or less than 0.99 or $99 \%$.

\section{Introduction}

Urbanization, consequent changes in pattern of human consumption, globalization of food market and climate change lead to new challenges against foodborne pathogens. Convenient, ready to eat foods provides a favourable atmosphere for toxin producing bacteria such as Staphylococcus aureus mainly due to inhibition of competitive microbial flora and post-process contamination. The European Food Safety Authority (EFSA) reported in 2009 that cheese followed by mixed or buffet meals were two main food vehicles involved in outbreaks of food poisoning caused by staphylococcal toxins. Scallan et al., (2011) also stated that $S$. aureus is a significant cause of foodborne diseases, causing an estimated 241,000 illnesses per year in United States and Shale et al., (2005) too observed that in developing countries Staphylococcus aureus 
has been consistently shown to be one of the most important micro-organisms responsible for food poisoning outbreaks. In India, there are no systematic studies to understand the types of foods involved and the etiological agent causing the foodborne diseases (Sudershan et al., 2014) which is mainly due to neglected surveillance, under reporting, poor consumer awareness about food safety issues. Consequently, the information on incidence of Staphylococcal Food Poisoning (SFP) and implicated foods are very limited. However, several studies have indicated the prevalence Staphylococcus aureus in foods and environment in general (Ghosh et al., 2007; Mahale et al., 2008; Tambekar et al., 2011; Agarwal et al., 2012) and chicken meat and its products in particular (Ruban et al., 2012; Arul Kumar and Saravanan, 2011; Badhe et al., 2013; Sudershan et al., 2014). Biryani is a rice-based mixed meal containing rice, spices, condiments and chicken pieces. One of the most popular, often consumed Prepare, Cook and Serve (PCS) meat preparation in the Indian subcontinent and however, the customary handling and storage practices in catering environments as well as households including eating habits of consumers pose a threat of staphylococcal intoxication.

In this context, Predictive Modeling (PM) and Quantitative Microbiological Risk Assessment (QMRA) are the promising tools where the former operate to fill the knowledge gaps by predicting the possible growth and multiplication of bacterial pathogens in different steps/modules of food supply chain and the latter to assess the consequent risk posed to the public health upon exposure (i.e., consumption), provided risk factors i.e., adequate information on food supply chain, especially, food processing and handling environment, pathogen behavior and incidences and consumption pattern of the food concerned are known. Hence, the present study was carried out to determine the possibility of Chicken Briyani serving as a source of risk to contract Staphylococcal Food Poisoning to the consumers, using predictive modeling and Quantitative Microbiological Risk Assessment, in the given context.

\section{Materials and Methods}

\section{Overview of modeling contamination of $S$. aureus in chicken briyani}

A QRAM for S. aureus in Chicken Briyani (Fig. 1) was constructed in an Excel spreadsheet and was simulated using @ risk (version 4.1, Palisade, Newfield, NY). The retail-to table pathway was modeled as a series of unit operations and associated pathogen events that included initial contamination of raw chicken at retail (node 1 ), growth during caterer transport (node 2), storage at catering establishments (node 3), thermal inactivation during cooking (node 4), post process contamination during serving (node 5; on the day consumption) and storage of leftover (node 6; consumption after overnight storage). This risk model explicitly assumes that all the Coagulase Positive Staphylococci in raw retail chicken (results from the previous study; $3.79 \log \mathrm{CFU} / \mathrm{g}$ ) are $S$. aureus and $95 \%$ of them carrying enterotoxigenic gene sequences. Further, this model assumes that all retail raw chickens were individually packaged and thus, cross contamination did not occur during caterer transport (node 2) and subsequent storage (node 3) and hence, the prevalence was same in node 1, 2 and 3.

This model also presumes that other ingredients added while cooking chicken /preparing chicken briyani (node 4) did not contain any $S$. aureus whereas post-process contamination occurred immediately after cooking (node 4) but before serving (node 5). 
The level of contamination and incidence of $S$. aureus in the latter node was defined by the data from an unpublished work on chicken briyani. An overview of the simulation variables and distributions that were used in this risk assessment model are discussed in more detail in input settings (2.1.2). In general, pathogen events were modeled using PERT, Triangular and Discrete distributions as well as predictive modeling software 'Combase' either singly or by linking these distributions to model rare pathogen events.

\section{Input settings}

\section{Node 1 - Initial contamination of raw chicken at retail}

The first node in the QRAM simulated the initial contamination of raw chicken with S.aureus at retail. The data from case study 1 on CPS count in retail chicken meat and prevalence were used to define input settings with necessary modifications as per our assumptions made earlier. The data on $S$. aureus count in retail raw chicken (initial contamination) were subjected to distribution fitting in @ risk. The best fitting distribution was chosen according to chi-square test, probability-probability plot, quantile-quantile plot and also by comparing the descriptive statistical parameters.

\section{Node 2 - Caterer transport}

The second node simulated growth of S.aureus on retail raw chicken. To establish input settings for this node, personal opinion was made on time (30 $\mathrm{min}, 120 \mathrm{~min}$ ) and temperature $\left(\min .23 .5^{\circ} \mathrm{C}, \max 35.3^{\circ} \mathrm{C}\right.$ ) data for caterers transport of raw chicken meat from retail stores to catering units and the same were used in the growth model. Uniform distribution was used to define time and temperature data.

\section{Node 3 - Storage at catering unit}

The third node simulated growth of $S$. aureus on retail raw chicken while the caterers store them for a while in the catering unit before cooking. To establish input setting for this period, again personal opinion was made on temperature $\left(\min 23.5^{\circ} \mathrm{C}, \max 35.3^{\circ} \mathrm{C}\right)$ and duration of storage $(\min 1 \mathrm{~h}, \max 3 \mathrm{~h})$ as there were no available data and the same were utilized in the growth model. As in the previous node, uniform distribution was used to define data (Fig. 2).

\section{Node 4 - Cooking of chicken briyani}

The fourth node simulated the thermal inactivation of $S$. aureus during preparation of Chicken Briyani (cooking of chicken). In this node, it was assumed that chicken briyani was cooked in the catering unit at a minimum temperature of $68^{\circ} \mathrm{C}$, maximum of $72^{\circ} \mathrm{C}$ and median of $70^{\circ} \mathrm{C}$ for a minimum duration of 45 min, maximum of $90 \mathrm{~min}$ and most likely of $60 \mathrm{~min}$. These time and temperature values were used to define the input settings for the PERT distributions in constructing the thermal inactivation model. Result of the cooking model simulation indicated that the $\log$ cycle reduction of $S$. aureus ranged from 266.17 to -137.02 with a median value of 189.39. These values were used to define the PERT distribution for cooking.

\section{Node 5 - Serving - on the day consumption}

The fifth node simulated post processcontamination of Chicken Briyani with $S$. aureus and its growth during serving. Here it was assumed that the contamination of chicken briyani was occurred immediately after cooking when left at ambient temperature for considerable period of time that would promote growth of $S$. aureus. To establish input setting for this period, again personal opinion was made on temperature 
(min $27^{\circ} \mathrm{C}$ and $\max .32^{\circ} \mathrm{C}$ ) and duration of storage (min $1 \mathrm{~h}$, most likely $2 \frac{1}{2} \mathrm{~h}$ and max. 8h) as there were no available data and the same were utilized in the growth model and thereby subjected to uniform and PERT distributions, respectively. With respect to level (initial) of post process-contamination and incidence of $S$. aureus, as mentioned earlier, the data from an unpublished work were used to establish input setting and discrete distribution was applied on incidence data (Fig. 3).

\section{Node 6 - storage of leftover (Consumption after overnight storage)}

The sixth node simulated growth of $S$. aureus in chicken briyani that has been kept overnight at refrigerated temperature in the households. To establish input setting for this period, again personal opinion was made on temperature $\left(\min 6^{\circ} \mathrm{C}\right.$; most likely $7.5^{\circ} \mathrm{C}$ and $\max 9^{\circ} \mathrm{C}$ ) and duration of storage $(\min 8 \mathrm{~h}$, most likely $10 \mathrm{~h}$ and $\max 11 \mathrm{~h}$ ) as there were no available data and the same were utilized in the growth model. Pert distribution was used to define data (Fig. 4).

\section{Exposure assessment}

S. aureus causes food borne diseases by the enterotoxins it produces. Several studies have shown that the concentration of $S$. aureus needed for detectable toxin production in a variety of foods varies between $10^{6}$ and $4 \times 10^{7}$ CFU/g (Gockler et al., 1988; Anunciacao et al., 1995; Walls and Scott, 1997; Lee et al., 2004; Fujikawa and Morozumi, 2006). According to the U.S. Food and Drug Administration, a toxin dose of less than 1.0 $\mu \mathrm{g}$ in contaminated food will produce symptoms of staphylococcal intoxication and this toxin level is reached when $S$. aureus populations exceed $10^{5} \mathrm{CFU} / \mathrm{g}$ (Food and Drug Administration, 1992). Based on all this information, $10^{5} \mathrm{CFU} / \mathrm{g}$ was used as the minimum concentration of $S$. aureus needed to cause foodborne disease, although further research might refine this value to a greater degree. In the present study, exposure assessment has been carried out in two nodes (Node 5 and 6).

\section{Probability of illness}

The probability of illness due to consumption of chicken briyani has been calculated by the exponential model proposed by the Rose and Haas (1999). The model proposed is as follows.

$\mathrm{P}$ (response) $=1-(-\mathrm{k} x$ dose $)$ where ' $\mathrm{k}$ ' is optimized parameter

\section{Simulation modeling}

The data, models and user inputs were entered into an Excel (Microsoft, Redmond, WA) spreadsheet. Modeling @ RISK software (Palisade Corporation) was used to perform Monte Carlo simulation of $\sim 10000$ iterations.

\section{Results and Discussion}

Estimated level of contamination at the point of consumption (using Combase predictive models and @ risk software)

The data on concentration of S.aureus, as per assumption made earlier, in retail raw chicken meat from previous study was utilized to demonstrate the distribution of the initial contamination level of S.aureus. The triangular distribution was chosen to define the extent of pathogen events (initial contamination) in node 1 . In $90 \%$ of the chicken meat samples the $S$. aureus count varied between 3.28 and $4.26 \mathrm{log} \mathrm{CFU} / \mathrm{g}$ of chicken and discrete distribution was used to define the incidence of $S$. aureus contamination. From these probabilistic distributions on extent of initial contamination 
and incidence, the output distribution at retail has been obtained. The maximum number of colonies obtained in output distribution at retail were approximately $2.87 \times 10^{4} \mathrm{CFU} / \mathrm{g}$ and the same was used to determine the logarithmic growth per hour (0.541 log $\mathrm{CFU} / \mathrm{h}$ ) using Combase growth model and extent of potential growth during the period of transport to catering unit (Node 2) was estimated to be about $0.68 \log \mathrm{CFU} / \mathrm{g}$ under given time and temperature conditions as well as incidence rate. Similarly, the maximum number of colonies obtained in the output distribution for node 2 (Transport to catering unit) was used to determine the output for node 3 (storage at catering unit).

As there was no suitable thermal inactivation model for S.aureus, both in Combase and Pathogen Modeling Program, the logarithmic reduction in the colonies during preparation of Chicken Briyani/cooking of chicken (node 4) was determined by mathematical calculation using $\mathrm{D}$-value (D70=0.33 min) under the given risk factors such as temperature and time. The results revealed that there would be approximately $193.45 \log$ $\mathrm{CFU} / \mathrm{g}$ reduction in $S$. aureus under given cooking time and temperature combination and consequently, no colonies could be identified during the end of cooking process (node 4). As per the assumption mentioned in 2.1, post-process contamination of Chicken Briyani occurred immediately after cooking (node 4) but before serving (node 5) with 3.35 $\log \mathrm{CFU} / \mathrm{g}$ of $S$. aureus (unpublished data). Based on the prevailing practices followed in the catering units, it was presumed that there would be growth of contaminating (postprocess) S.aureus during the time delay between cooking and serving and hence the same was predicted using Combase growth model. The output distribution for serving based on cross contaminating microorganisms and its growth was calculated. Similarly, output distribution was calculated for node 6 under given temperature - time conditions to determine the pathogen event.

\section{Exposure per serving}

As mentioned in section 2.3 exposure assessment has been carried out in two nodes (Node 5 and 6). Further, $10^{5} \mathrm{CFU} / \mathrm{g}$ was used as the minimum concentration of $S$. aureus needed to cause foodborne disease. At node 5 (on the day consumption), $63.33 \%$ of the population consumes less than or equal to $10^{5}$ cfu/serve. Conversely, $36.67 \%$ of the population is at risk of staphylococcal food intoxication. From this exposure assessment study, it could be inferred from the detailed statistics that $50 \%, 90 \%, 95 \%$ and $99 \%$ of the population had the exposure level of equal to or less than $0.00,8.54,8.82$ and $12 \log$ cfu/serve, respectively when exposure assessment was made at Node 5. Surprisingly, similar percentile of the population consumes less than or equal to $10^{5} \mathrm{cfu} /$ serve at Node 6 . However, the detailed statistics revealed that $50 \%, 90 \%, 95 \%$ and $99 \%$ of the population had the exposure level of equal to or less than $0.00,12,12$ and $13 \log$ cfu, respectively when exposure assessment was made at Node 6 . This finding is concordance with Sudershan et al., 2014 who reported that chicken briyani with Staphylococcus aureus count of 8.5$9.5 \times 10^{8} \mathrm{cfu} / \mathrm{g}$ involved in a food poisoning outbreak in Hyderabad city, Andhra Pradesh.

\section{Estimation of probability of illness}

From the cumulative distribution and descriptive statistics, it could be inferred that in $50 \%$ of the cases, the probability of risk in getting ill to at least one recipient is equal to or less than 0.00 or $0 \%$. In $95 \%$ of the cases, the probability of getting illness due to the consumption of $S$. aureus contaminated Chicken Briyani to at least one recipient is equal to or less than 0.99 or $99 \%$. In $99 \%$ of the cases, the probability of getting illness to 
at least one recipient is equal to or less than 1 or $100 \%$ at node 5 . This study reveals the higher probability of illness due to Staphylococcal Food Poisoning in consumers of Chicken Briyani. The time delay between preparation and consumption as well as temperature of storage are the important risk factors (Fig. 5).

Fig.1 Distribution of initial contamination (extent) of retail raw chicken with $S$. aureus

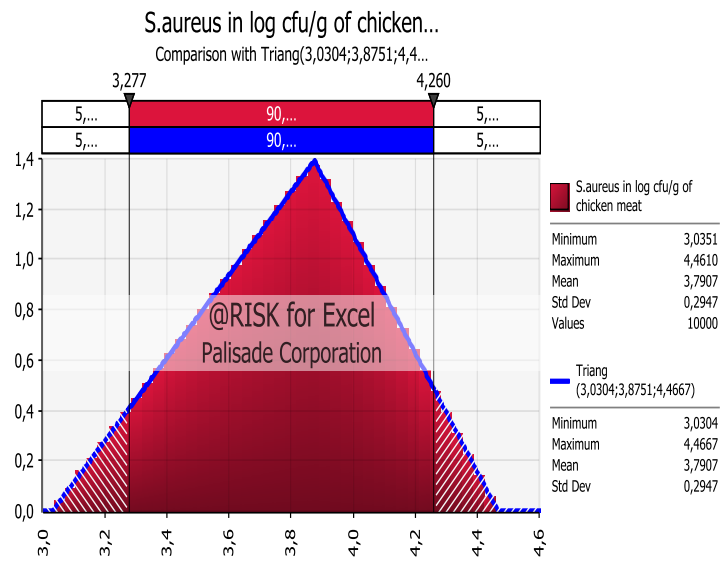

Fig.2 Prevalence distribution for nodes $1,2 \& 3$

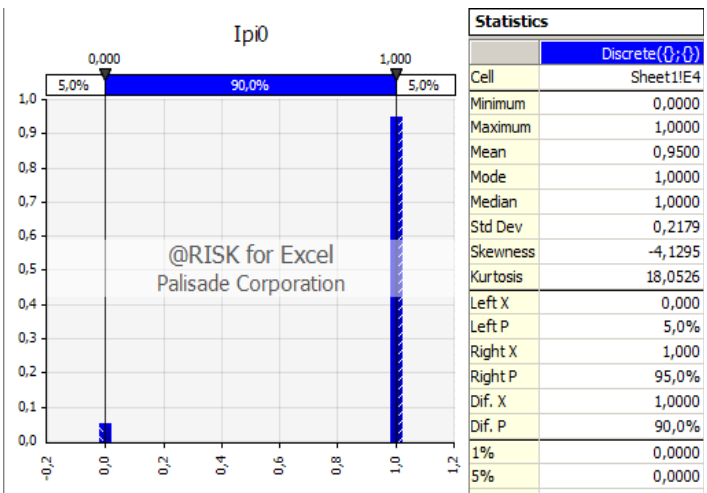

Fig.3 Exposure/serve or week at Node 5

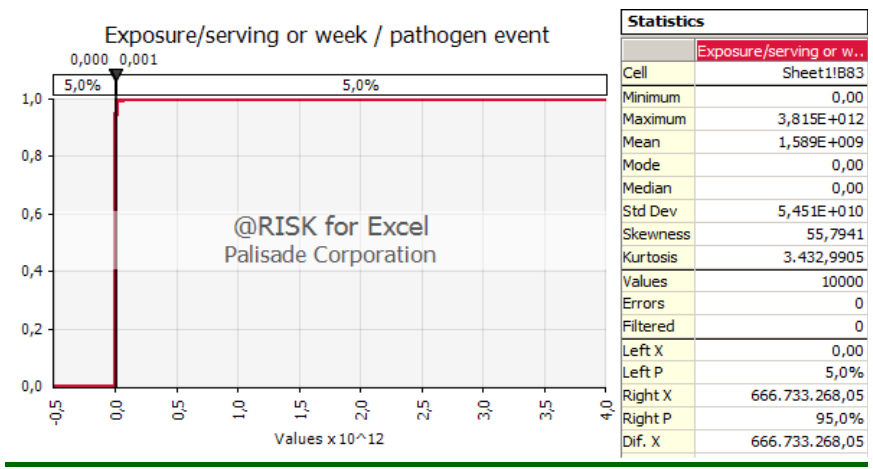


Fig.4 Exposure/serve or week at Node 6

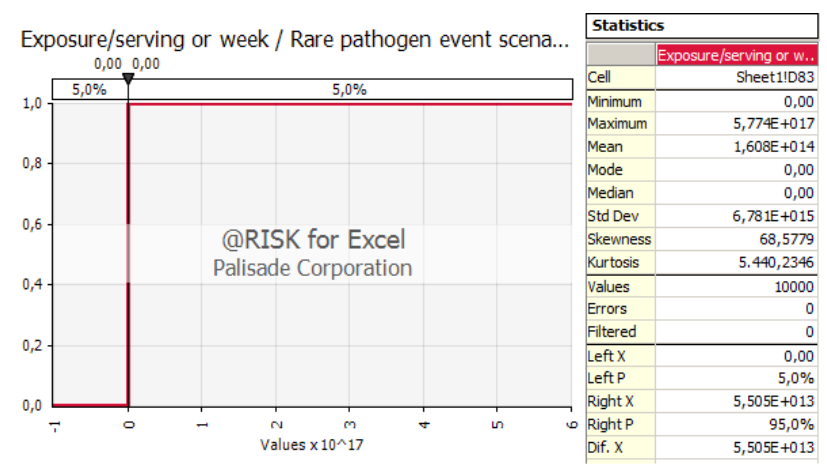

Fig.5 Estimation of probability of illness

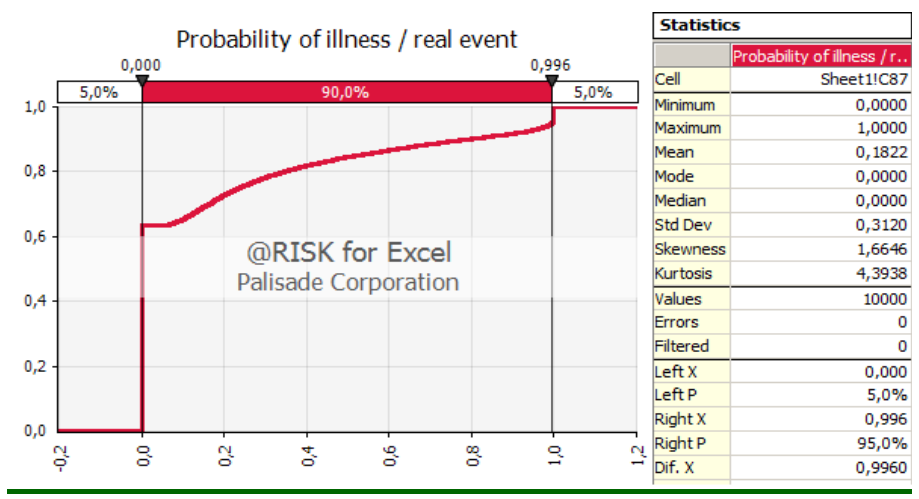

In conclusion, initial microbial load in raw chicken, transport and storage at ambient temperature significantly influence the microbial load of the chicken before cooking.

Cooking is a crucial event which should be monitored to avoid happening of untoward events.

Post-process contamination and eating habits appears to important factors makes the population vulnerable to SFP.

\section{Acknowledgement}

I gratefully acknowledge the support and generosity of ITP Food Safety Core group, Faculty of Biosciences Engineering, Gent University, Belgium and VLIR UOS without which the present study could not have been completed.

\section{References}

Agarwal, A., Awasthi, V., Dua, A., Ganguly, S., Garg, V. and S.S. Marwaha, 2012. Microbiological Profile of Milk: Impact of Household Practices. Ind. J. Public Health, 56(1): 88-94.

Anunciacao, L.L.C., Linardi, W.R., Do Carmo, L.S., Bergdoll, M.S., 1995. Production of staphylococcal enterotoxin A in cream-filled cake. Int. J. Food Microbiol. 26, 259-263.

Arul Kumar, T. and S. Saravanan, 2011. Assessment of contamination in chicken meat by food- borne Staphylococcus aureus. Intl. J. Res. Pure and Appl. Microbiol, vol. 1:59-60.

Badhe, S.R., Fairoze, M. N. and S. Sudarshan, 2013. Prevalence of Food borne pathogens in market samples of chicken meat in Bangalore, India. Ind. J. Anim. 
Res., 47(3): 262-264.

Food and Drug Administration, 1992. Foodborne Pathogenic Microorganisms and Natural Toxins

Fujikawa, H., Morozumi, S., 2006. Modeling Staphylococcus aureus growth and enterotoxin production in milk. Food Microbiol. 23, 260-267.

Ghosh, M. Wahi, S., Kumar, M. and A. Ganguli, 2007. Prevalence of enterotoxigenic Staphylococcus aureus and Shigella spp. in some raw street vended Indian foods, Intl. J of Environ., Hlth., Res., 17(2): 151-156.

Gockler, L., Notermans, S., Kramer, J., 1988. Production of enterotoxins and thermonuclease by Staphylococcus aureus in cooked egg-noodles. Int. J. Food Microbiol. 6, 127-139.

Lee, H., Lee, G., Yoon, E., Kim, H., Kang, Y., Lee, D., Park, J., Lee, S., Woo, G., 2004. Computation of maximum edible time using monitoring data of Staphylococcus aureus in kimbab and food Micro Model. J. Food Hyg. Saf. $19,49-54$.

Mahale, D.P., Khade, R.G., and V.K. Vaidya, 2008. Microbiological Analysis of Street Vended Fruit Juices from Mumbai City, India. Internet $\mathbf{J}$ of Food Safety, 10:31-34.

Ruban, S.W., Prabhu, N. K. and G. S. Naveen Kumar, 2012. Prevalence of food borne pathogens in market samples of chicken meat in Bangalore, Int. Food Res. J, vol. 19:1763-1765.

Rose, J.B., and Haas, C.N., 1999. A risk assessment framework for the evaluation of skin infections and the potential impact of antibacterial soap washing. American Journal of Infection Control 27(6), S26-S33.

Scallan, E., Hoekstra, R. M., Angulo, F. J., Tauxe, R. V., Widdowson, M.A., Roy, S. L., Jones, J.L. and P.M. Griffin, 2011. Foodborne illness acquired in the United States - Major pathogens. Emerging Infectious Diseases, 17(1):7.

Shale, K., Lues, J.F.R., Venter, P and E.M. Buys, 2005. The distribution of Staphylococcus sp. on bovine meat from a battoir deboning rooms. Food Microbiol., 22, pp 433-438.

Sudershan, R. V., Naveen Kumar, R., Kashinath, L., Bhaskar, V and K. Polasa, 2014. Foodborne Infections and Intoxications in Hyderabad India. Epidemiology Res. Intl., vol 2014:1- 5.

Tambekar, D. H., Kulkarni, R.V., Shirsat, S.D. and D.G. Bhadange, 2011. Bioscience Discovery, 2(3): 350-354.

Walls, I., and Scott, V.N., 1997. Use of predictive microbiology in microbial food safety risk assessment. Int. J. Food Microbiol. 36, 97-102.

\section{How to cite this article:}

Selvan, P. 2019. Quantitative Exposure Assessment of Enterotoxin-Producing Staphylococcus aureus in Chicken Briyani. Int.J.Curr.Microbiol.App.Sci. 8(04): 2505-2512. doi: https://doi.org/10.20546/ijcmas.2019.804.291 